\title{
CHAOS CONTROL OF A MODIFIED 4-D MEMRISTOR CHAOTIC OSCILLATOR VIA PASSIVE CONTROL TECHNIQUE
}

\author{
M. I. Babalola ${ }^{1}{ }^{*}$, B. E. Iyorzor ${ }^{2}$ and I. Odesanya ${ }^{3}$ \\ 1, 2 Department of Physics, University of Benin, Benin City, Edo State. NIGERIA \\ 3 DePARTMENT OF Physics, FEDERAl University, LOKOJA, Kogi STATE, NIGERIA \\ E-mail addresses:1 michael.babalola@uniben.edu, ${ }^{2}$ beniyorzor@uniben.edu, \\ 3ituabhor.odesanya@fulokoja.edu.ng
}

\begin{abstract}
A modified memristor chaotic oscillator is proposed for a class of four dimensional chaotic systems. This modification can serve as an alternative way of designing the memristor circuit. We used the passive control technique which requires a single controller to stabilize the system which is presented in our numerical simulations for validation. Our results show that the passive control technique is a very effective technique for controlling chaotic systems.
\end{abstract}

Keywords: Chaos; passive control; memristor; controllers; chaotic oscillator; nonlinear circuit

\section{INTRODUCTION}

Chaos is a phenomenon which has been found to be present in many fields of studies (they include physics, biology, chemistry, etc.) and in nature (which include weather, earthquake, etc). This chaotic behavior in dynamical systems was first studied by Lorenz [1] who developed a set of three-dimensional autonomous ordinary differential equations which he used to model the convection in the atmosphere. Since then other chaotic systems have been developed which include Rossler system [2], Chen system [3], Lu system [4] and many other systems [5-10]. These chaotic systems can be very useful in nonlinear circuit [11], communication security [12] and many other areas. Despite the fact that there are many chaotic systems that have been proposed, there are still new chaotic systems that are been proposed [13-15].Chaos control is a phenomenon in nonlinear dynamics which could mean suppression of a chaotic system or enhancement of a chaotic system. The idea of chaos control was introduced by a group of researchers [16], and since then it has attracted the attention of many researchers. In chaos control, depending on the technique used, controllers are designed which when introduced to the chaotic system will asymptotically and globally stabilize the system. In this work, we present a memristor chaotic oscillator [12] which has been studied extensively [17] and a modified form of the memristor chaotic oscillator. We also adopt the passive control technique [18-20] to stabilize the modified system. A memristor is a device in an electrical circuit that controls the flow of electrical current and remembers the amount of charge that has previously flowed through it. Memristors are important because they are non-volatile, meaning that they retain memory without power.

The reason for the choice of technique is because it requires a single controller to stabilize the entire system unlike other techniques which require three controllers.

\section{SYSTEM DESCRIPTION}

The memristor chaotic oscillator is defined by [12]:

$$
\left\{\begin{array}{l}
\dot{x}=a y+\beta x-\gamma x p^{2} \\
\dot{y}=x-y+z \\
\dot{z}=-\delta y-\sigma z \\
\dot{p}=x
\end{array}\right.
$$

where $\alpha, \beta, \gamma, \delta$ and $\sigma$ are parameters that make the system chaotic when $\alpha=16.4, \beta=3.28, \gamma=19.7, \delta=15$ and $\sigma=0.5$.

The chaotic attractor of system (1) is shown in Fig. 1. The modified memristor chaotic oscillator is defined by:

$$
\left\{\begin{array}{l}
\dot{x}=a y-\beta x-\gamma x p^{2} \\
\dot{y}=x-y+z \\
\dot{z}=-\delta y-\sigma z \\
\dot{p}=x
\end{array}\right.
$$




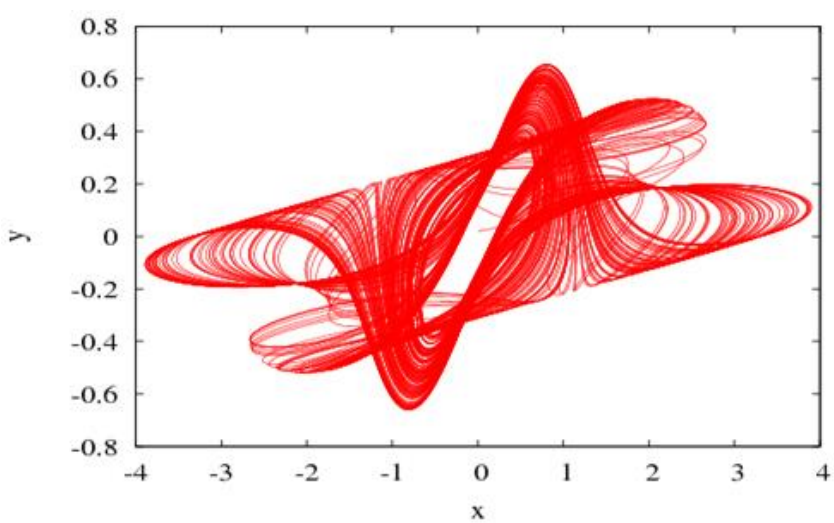

Figure 1: 2D attractor for the memristor chaotic oscillation.

We observed that system (2) exhibit the same chaotic attractor when $\alpha=16.4, \beta=3.28, \gamma=19.7, \delta=15$ and $\sigma=0.5$ and also when $\alpha=8.2, \beta=1.64, \gamma=9.85, \delta=7.5$ and $\sigma=0.25$ as can be seen in Fig. 2

\section{THEORY OF PASSIVE CONTROL}

Considering system (3) which is modeled by a differential equation with input vector $u(t)$ and $a$ corresponding output vector $\mathrm{y}(\mathrm{t})$

$$
\left\{\begin{array}{l}
\dot{x}=f(x)+g(x) u \\
y=h(x)
\end{array}\right.
$$

where $x \in R^{n}$ is a state variable, the input $u \in U$ and output $y \in R^{m} . f(x)$ and $g(y)$ are smooth vector fields. $\mathrm{h}(\mathrm{x})$ is a smooth mapping. System (3) is said to be passive as long as it meets the following condition:

For any $t \geq 0$ there is always a real constant $\beta$ which satisfies the inequality

$$
\int_{0}^{t} u^{T}(\tau) y(\tau) d \tau \geq \beta
$$

or there exist real constant $\beta$ and $\rho>0$ that satisfy the inequality

$$
\int_{0}^{t} u^{T}(\tau) y(\tau) d \tau+\beta \geq \int_{0}^{t} \rho u^{T}(\tau) y(\tau) d \tau
$$

If the system has been made passive, then the control parameter will asymptotically stabilize to the equilibrium $x=0$ of the system (1). If $z=\theta(x)$, then system (3) can be transformed into

$$
\left\{\begin{array}{l}
\dot{z}=f_{0}(z)+P(z, y) y \\
\dot{y}=b(z, y)+a(z, y) u
\end{array}\right.
$$

If system (3) has a relative degree $[1, \ldots 1]$ at $\mathrm{x}=0$, and is a minimum phase, system (6) will be said to be passive and will achieve stability asymptotically at an equilibrium point with the help of a state feedback control law:

$u=a(z, y)^{-1}\left[-b^{T}(z, y)-\frac{\partial}{\partial z} W(z) P(z, y)-k y+v\right]$ where $W(z)$ is a lyapunov function, $\alpha$ is a positive real

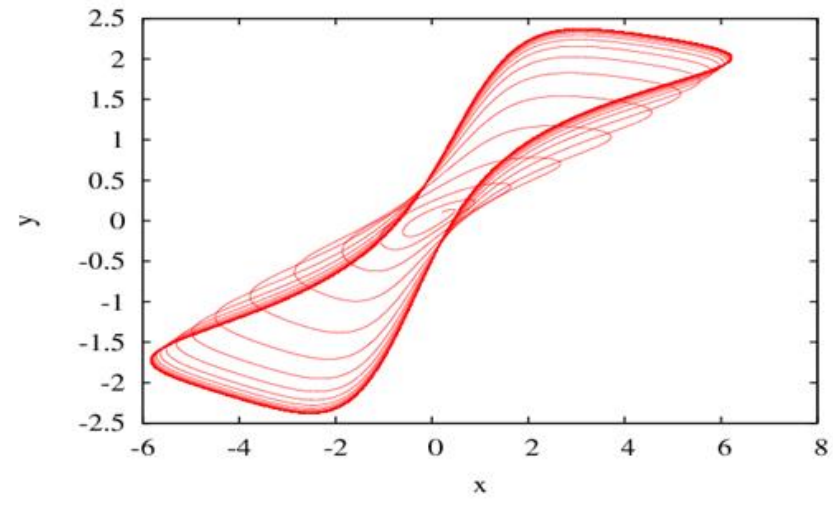

Figure 2: 2D attractor for the modified memristor chaotic oscillation.

value and $v$ is an external signal that is connected to the reference input.

\section{CONTROLLING THE MODIFIED SYSTEM}

Considering system (2), if we put $\mathrm{x}=\mathrm{z}_{1}, \mathrm{z}=\mathrm{z}_{2}, \mathrm{p}=\mathrm{z}_{3}$, and leaving $y$ unchanged then

$$
\left\{\begin{array}{l}
\dot{z}_{1}=a y-\beta z_{1}-\gamma z_{1} z_{3}^{2} \\
\dot{z}_{2}=-\delta y-\sigma z_{2} \\
\dot{z}_{3}=z_{1} \\
\dot{y}=z_{1}-y+z_{2}+u
\end{array}\right.
$$

Comparing system (6) with system (7) we have

$$
\left\{\begin{array}{l}
f_{0}(z)=\left[\beta z_{1}-\gamma z_{1} z_{3}^{2}-a z_{2} z_{1}\right]^{T} \\
P(z, y)=[\alpha-\delta]^{T} \\
b(z, y)=z_{1}-y+z_{2} \\
\alpha(z, y)=1
\end{array}\right.
$$

In designing the control parameter once the system has been made passive, we choose a function of the form: $V(z, y)=W(z)+\frac{1}{2} y^{2}$ where $\mathrm{W} \quad(\mathrm{z})$ is Lyapunov function of $f_{0}(\mathrm{z}) W(z)=\frac{1}{2} z_{1}{ }^{2}+\frac{1}{2} z_{2}{ }^{2}+z_{3}{ }^{2}$

Once the dynamics of system (6) is zero, then it is said to describe those internal dynamics which are consistent with external constraint $\mathrm{y}=0$

We have $z=f_{o}(z)$ and

$$
\frac{d}{d t} W(z, y)=\frac{d}{d z} W(z) \dot{z}+y \dot{y}
$$

$\frac{d}{d t} W(z)=\frac{\partial}{\partial z} W(z) \dot{z}=\left[z_{1} z_{2} z_{3}\right]\left[\begin{array}{l}-\beta z_{1}-\gamma z_{1} z_{3}^{2} \\ -a z_{2} \\ z_{1}\end{array}\right]$

$=-\beta z_{1}^{2}-a z_{2}^{2}-\left(y z_{1}^{2} \beta z_{3}^{2}\right)$ it is obvious that $W(z)$

$>0$ and $\dot{W}$

$\leq 0$, and putting system (6)into (8)we have $\frac{d}{d t} V(z, y)$

$=\frac{\partial}{\partial z} W(z) f_{0}(z)+\frac{\partial}{\partial z} W(z) P(z, y) y+y b(z, y)$

$+y a(z, y) u$

Since system (6) is a minimum phase, then we have $\frac{\partial}{\partial z} W(z) f_{o}(z) \leq 0$ and 


$$
\begin{aligned}
\frac{d}{d t} V(z, y) \leq \frac{\partial}{\partial t} W & (z) P(z, y) y+y b(z, y) \\
& +y\left[-b^{T}(z, y)-\frac{\partial}{\partial t} W(z) P(z, y)-k y\right. \\
& +v] \\
& \frac{d}{d t} V(z, y) \leq-\alpha y^{2}+y v
\end{aligned}
$$

Integrating both sides of Eq. (11) we have

$$
V(z, y)-V\left(z_{0}, y_{o}\right) \leq \int_{0}^{t} \alpha y(\tau)^{2} d \tau+\int_{0}^{t} v(\tau) y(\tau) d \tau
$$

If $V(z, y) \geq 0$ and setting $V\left(z_{0}, y_{0}\right)=\mu$ when Eq. (12) is rearranged we have

$$
\begin{aligned}
\int_{0}^{t} v(\tau) y(\tau) d \tau+\mu & \geq \int_{0}^{t} \alpha y(\tau)^{2} d \tau+V(z, y) \\
& \geq \int_{0}^{t} \alpha y(\tau)^{2} d \tau
\end{aligned}
$$

Since Eq. (13) satisfies the condition earlier stated, then the system (6) is said to be a passive system. Substitute Eq. (7) into the control parameter $u$ we :

$$
u=\left[z_{1}(-1-\alpha)+z_{2}(\delta-1)+y(1-k)+v\right]
$$

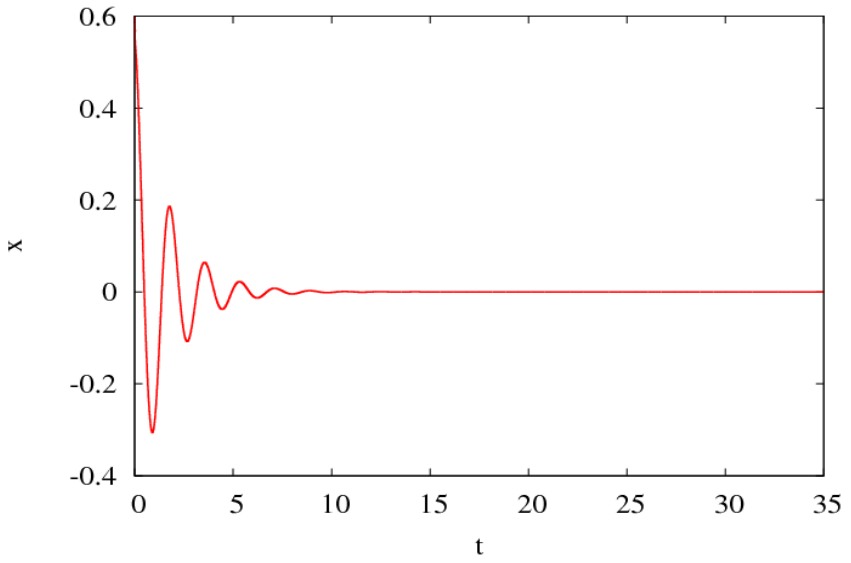

Figure 3: Time series for the first state of the modified memristor chaotic oscillation when controller is activated.

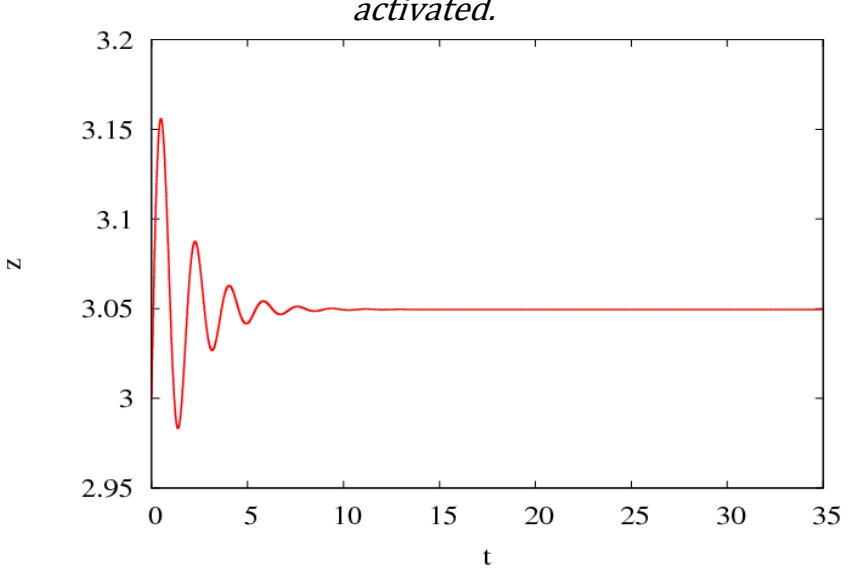

Figure 5: Time series for the third state of the modified memristor chaotic oscillation when controller is activated.

\section{NUMERICAL SIMULATION}

Some numerical simulations are presented in order to verify the performance of the control parameter. In the simulations, the initial conditions are set to $x(0)=1$ $, y(0)=4, \mathrm{z}(0)=3, \mathrm{p}(0)=6$, respectively. The fourthorder Runge-Kutta method is used to solve the differential equations (1), (2) and (7) with time step size of 0.001in all numerical simulations. In Figs 3, 4, 5 and 6 , we observed that the modified system has successfully been controlled.

\section{CONCLUSION}

In summary, we have stabilized the proposed modified memristor chaotic oscillator using the passive control technique which we found to be effective from numerical simulation. This control technique requires a single control parameter to stabilize the entire system unlike other control techniques that require three control parameters.

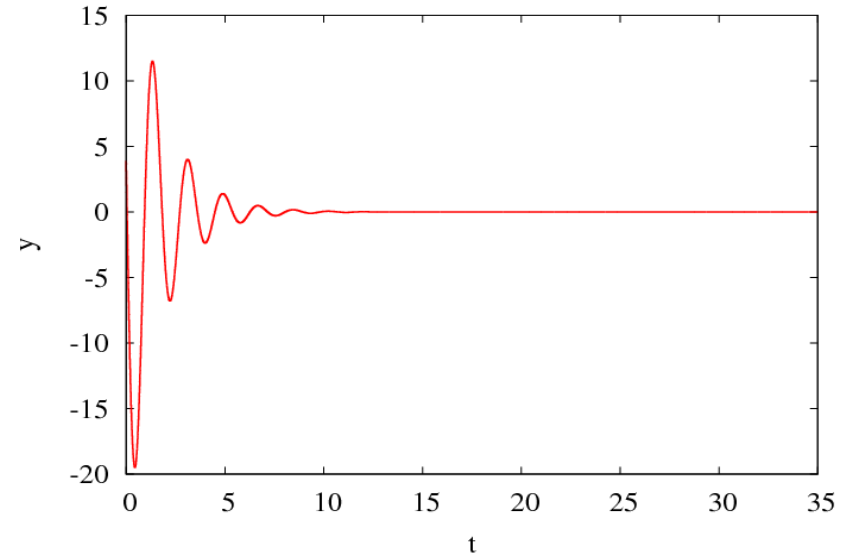

Figure 4: Time series for the second state of the modified memristor chaotic oscillation when controller is activated.

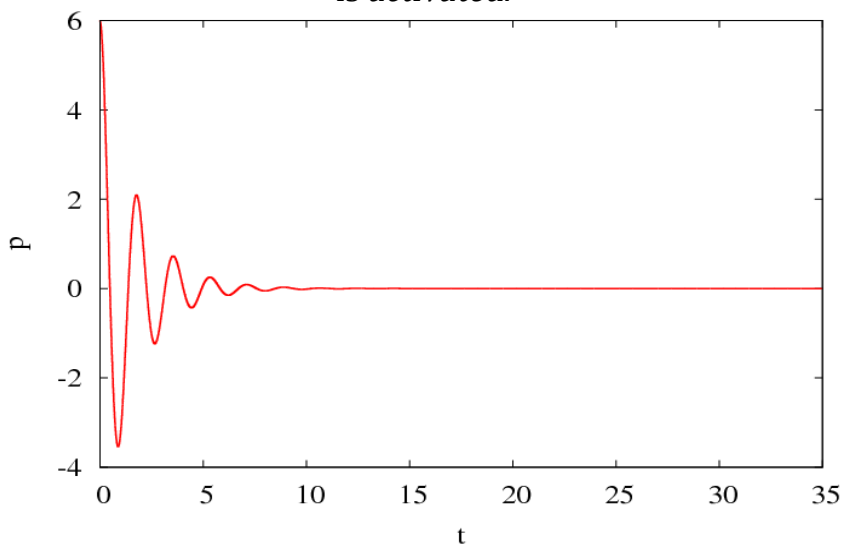

Figure 6: Time series for the fourth state of the modified memristor chaotic oscillation when controller is activated. 


\section{REFERENCES}

[1] Lorenz, E. N. "Deterministic nonperiodic flow." Journal of the atmospheric sciences Vol. 20, Number 2, pp130 - 141, 1963.

[2] Rössler, O. E. "An equation for continuous chaos." Physics Letters A, Vol. 57, Number 5, pp.397 398, 1976.

[3] Chen, G., and Ueta T. "Yet another chaotic attractor." International Journal of Bifurcation and chaos, Vol. 9, Number 7, pp.1465 - 1466, 1999.

[4] Lü, J., and Chen G. "A new chaotic attractor coined." International Journal of Bifurcation and chaos, Vol. 12, Number 3, pp. 659 - 661, 2002.

[5] Genesio, R., and Tesi A. "Harmonic balance methods for the analysis of chaotic dynamics in nonlinear systems." Automatica Vol. 28, Number 3, pp. $531-548,1992$.

[6] Tigan, G., and Opriș D. "Analysis of a 3D chaotic system." Chaos, Solutions \& Fractals Vol. 36, Number 5, pp1315-1319, 2008.

[7] Rikitake, T. "Oscillations of a system of disk dynamos." In Mathematical Proceedings of the Cambridge Philosophical Society, Vol. 54, Number 1, pp. 89-105. Cambridge University Press, 1958.

[8] Belykh, V. N., and L. O. Chua. "New type of strange attractor from a geometric model of Chua's circuit." International Journal of Bifurcation and Chaos Vol. 2, Number 3, pp. 697 - 704, 1992.

[9] Chen, H. K., and Lee C. I. "Anti-control of chaos in rigid body motion." Chaos, Solitons \& Fractals Vol. 21, Number 4, pp. 957 - 965, 2004.

[10] Pikovski A. S., Rabinovich M. I., Trakhtengerts V. Y.: Onset of stochasticity in decay confinement of parametric instability, Sov. Phys. JETP Vol. 47, pp. $715-719,1978$.

[11] Pikovskii, A. S., M. I. Rabinovich, and V. Yu Trakhtengerts. "Onset of stochasticity in decay confinement of parametric instability." Sov. Phys.-
JETP (Engl. Transl.); Vol. 47, Number 4, pp. 715 720, 1978.

[12] Sun, J., Y. Shen, Q. Yin, and C. Xu. "Compound synchronization of four memristor chaotic oscillator systems and secure communication." Chaos: An Interdisciplinary Journal of Nonlinear Science, Vol. 23, Number 1, pp. 13140 - 13140, 2013.

[13] Lü, J., G. Chen, and D. Cheng. "A new chaotic system and beyond: the generalized Lorenz-like system." International Journal of Bifurcation and Chaos Vol. 14, Number 05, pp1507-1537, 2004.

[14] Qi, G., G. Chen, M. Antonie van Wyk, B. J. van Wyk, and Y. Zhang. "A four-wing chaotic attractor generated from a new 3-D quadratic autonomous system." Chaos, Solitons \& Fractals, Vol. 38, Number 3, pp. 705 - 721, 2008.

[15] Kim, D., and P. H. Chang. "A new butterfly-shaped chaotic attractor." Results in Physics Vol. 3, pp. 14 $-19,2013$.

[16] Ott, E., C. Grebogi, and J. A. Yorke. "Controlling chaos" Physical review letters Vol. 64, Number 11, pp. 1196 - 1199, 1990.

[17] Zhang, B., and F. Deng. "Double-compound synchronization of six memristor-based Lorenz systems." Nonlinear Dynamics Vol. 77, Number 4, pp. 1519 - 1530, 2014.

[18] Byrnes, C. I., and A. Isidori. "Asymptotic stabilization of minimum phase nonlinear systems." IEEE Transactions on Automatic Control Vol. 36, Number 10, pp. 1122 - 1137, 1991.

[19] Yu, W. "Passive equivalence of chaos in Lorenz system." IEEE Transactions on Circuits and Systems I: Fundamental Theory and Applications Vol. 46, Number 7, pp. 876 - 878, 1999.

[20] Qi, D., G. Zhao, and Y. Song. "Passive control of Chen chaotic system." In Intelligent Control and Automation, 2004. WCICA 2004. Fifth World Congress on, IEEE, Vol. 2, pp. 1284 - 1286, 2004. 